\title{
Modeling COVID-19 with Human Pluripotent Stem Cell-Derived Cells Reveals Synergistic Effects of Anti-inflammatory Macrophages with ACE2 Inhibition Against SARS-CoV-2
}

Fuyu Duan

School of Medicine, Tsinghua University

Liyan Guo

Prenatal Diagnostic Centre and Cord Blood Bank, Guangzhou Women and Children's Medical Center, Guangzhou Medical University

Liuliu Yang

Department of Surgery, Weill Cornell Medicine

Yuling Han

Department of Surgery, Weill Cornell Medicine

Abhimanyu Thakur

The Pritzker School of Molecular Engineering, the University of Chicago

Benjamin E. Nilsson-Payant

Department of Microbiology, Icahn School of Medicine at Mount Sinai

Pengfei Wang

Aaron Diamond AIDS Research Center, Columbia University Irving Medical Center

Zhao Zhang

Department of Medicine, Li Ka Shing Faculty of Medicine; the University of Hong Kong

Chui Yan Ma

Department of Medicine, Li Ka Shing Faculty of Medicine; the University of Hong Kong

Xiaoya Zhou

Prenatal Diagnostic Centre and Cord Blood Bank, Guangzhou Women and Children's Medical Cent

Teng Han

Sandra and Edward Meyer Cancer Center, Department of Medicine, Weill Cornell Medicine

Tuo Zhang

Genomic Resource Core Facility, Weill Cornell Medicine

Xing Wang

Genomic Resource Core Facility, Weill Cornell Medicine

Dong Xu

Genomic Resource Core Facility, Weill Cornell Medicine

Xiaohua Duan

Department of Surgery, Weill Cornell Medicine

Jenny Xiang

Genomic Resource Core Facility, Weill Cornell Medicine

Hung-fat Tse

Department of Medicine, Li Ka Shing Faculty of Medicine; the University of Hong Kong

Can Liao

Prenatal Diagnostic Centre and Cord Blood Bank, Guangzhou Women and Children's Medical Center, Guangzhou Medical University

Weiren Luo

Department of Pathology, The Second Affiliated Hospital of Southern University of Science and Technology

Fang-Ping Huang

Institute for Advanced Study (IAS), Shenzhen University

Ya-Wen Chen 
Department of Medicine, Hastings Center for Pulmonary Research, Division of Pulmonary, Critical Care and Sleep Medicine

\section{Todd Evans}

Department of Surgery, Weill Cornell Medicine

\section{Robert E. Schwartz}

Division of Gastroenterology and Hepatology, Department of Medicine, Weill Cornell Medicine

\section{Benjamin tenOever}

Department of Microbiology, Icahn School of Medicine at Mount Sinai

\section{David D. Ho}

Department of Microbiology, Icahn School of Medicine at Mount Sinai

\section{Shuibing Chen}

Department of Surgery, Weill Cornell Medicine

\section{Jie Na ( $\sim$ jie.na@tsinghua.edu.cn )}

School of Medicine, Tsinghua University

\section{Qizhou Lian ( $\nabla$ qzlian@hku.hk)}

Department of Medicine, Li Ka Shing Faculty of Medicine; the University of Hong Kong

\section{Huanhuan Joyce Chen ( $\sim$ joycechen@uchicago.edu )}

The Pritzker School of Molecular Engineering, the University of Chicago

\section{Short Report}

Keywords: COVID-19, SARS-CoV-2, Human pluripotent stem cell, Macrophages, disease modeling

Posted Date: September 15th, 2020

DOI: https://doi.org/10.21203/rs.3.rs-62758/v2

License: @ (7) This work is licensed under a Creative Commons Attribution 4.0 International License. Read Full License 


\section{Abstract}

Dysfunctional immune responses contribute critically to the progression of Coronavirus Disease-2019 (COVID-19) from mild to severe stages including fatality, with pro-inflammatory macrophages as one of the main mediators of lung hyper-inflammation. Therefore, there is an urgent need to better understand the interactions among SARS-CoV-2 permissive cells, macrophage, and the SARS-CoV-2 virus, thereby offering important insights into new therapeutic strategies. Here, we used directed differentiation of human pluripotent stem cells (hPSCs) to establish a lung and macrophage co-culture system and model the host-pathogen interaction and immune response caused by SARS-CoV-2 infection. Among the hPSC-derived lung cells, alveolar type II and ciliated cells are the major cell populations expressing the viral receptor ACE2 and co-effector TMPRSS2, and both were highly permissive to viral infection. We found that alternatively polarized macrophages (M2) and classically polarized macrophages (M1) had similar inhibitory effects on SARS-CoV-2 infection. However, only M1 macrophages significantly up-regulated inflammatory factors including IL-6 and IL-18, inhibiting growth and enhancing apoptosis of lung cells. Inhibiting viral entry into target cells using an ACE2 blocking antibody enhanced the activity of M2 macrophages, resulting in nearly complete clearance of virus and protection of lung cells. These results suggest a potential therapeutic strategy, in that by blocking viral entrance to target cells while boosting anti-inflammatory action of macrophages at an early stage of infection, M2 macrophages can eliminate SARS-CoV-2, while sparing lung cells and suppressing the dysfunctional hyper-inflammatory response mediated by M1 macrophages.

\section{Introduction}

The infection of severe acute respiratory syndrome coronavirus 2 (SARS-CoV-2) has already caused more than 5.4 million Coronavirus Disease-2019 (COVID-19) cases internationally (https://google.org/crisisresponse/covid19-map). Most COVID-19 patients show mild to moderate symptoms of fever, dry cough, fatigue and diarrhea, however, approximately $15 \%$ of confirmed cases progress to severe pneumonia, acute respiratory distress syndrome (ARDS) or multi-organ failure (Guan et al., 2020). The progression from mild to severe disease or death is principally attributed to dysfunctional immune responses (Mehta et al., 2020; Wang et al., 2020) together with viral damage of target cells. Given the lack of an effective vaccine or medication, a thorough understanding of immunological features caused by SARS-CoV-2 is critically important for studying viral pathobiology and therapeutic development.

Alveolar macrophages (AMs) are key sentinel cells for host defense in the respiratory system, producing cytokines and chemokines that are crucial components of innate immunity and mediators of immunopathology (Allard et al., 2018). The polarization of macrophages confers a heterogeneous function and plasticity depending on the duration of stimulation and microenvironment, which are discrete phenotypes associated with different inflammatory responses, typically termed the $\mathrm{M} 1 \varphi$ /pro-inflammatory and M2 $\varphi$ /anti-inflammatory macrophages (Gomez Perdiguero et al., 2015; Wynn et al., 2013). The distinction is known to be oversimplified, with macrophage dynamic activities spread along the M1-M2 phenotypic spectrum(Bian, 2020; Shapouri-Moghaddam et al., 2018). However, in general, $M 1 \varphi$ destroys pathogens by producing a large number of pro-inflammatory cytokines such as IL-1 $1 \beta$, TNFa, IL -6 and IL 18. In contrast, M2 $\varphi$ exhibits higher activity in phagocytosis against pathogens and for anti-inflammation (Mills, 2015; Murray, 2017). Recent studies (Liao et al., 2020; Xu et al., 2020) on immunity of COVID-19 patients indicate that the cells damaged by SARS-CoV-2 infection induced innate inflammation in the lungs that is largely mediated by pro-inflammatory macrophages and granulocytes. In addition to local damage, the pro-inflammatory macrophages release cytokines/chemoattractants and prime adaptive immune cell responses, which in some cases lead to dysfunctional immune responses and cytokine storm, followed by respiratory and even multi-organ failure (Xu et al., 2020). These studies imply a crucial role for macrophages in the progress of SARS-CoV-2 infection; a deeper understanding of the interactions among targeted cells, macrophages and SARS-CoV-2 could offer new ideas to help combat this deadly contagious disease.

The current most widely used model for SARS-CoV-2 research is the African green monkey derived Vero cells, which are very limited for modeling human disease. Although primary macrophages are more functionally or phenotypically representative of native macrophages in the tissue from which they are derived, they are difficult to obtain, proliferate slowly, and are often poorly characterized (Jobe et al., 2017). In this study, we generated lung cells and macrophages paired from the same cell origin, human pluripotent stem cell (hPSC) lines. This strategy overcomes a common concern about histocompatibility when studying human immune cells with other cell types, and provides theoretically unlimited cell resources for reliably modeling and studying immunology of macrophages and human lungs during SARS-CoV-2 infection. Our results using this platform demonstrate a potential therapeutic strategy through a combination of boosting anti-inflammatory macrophages and intervention of viral entry, to control SARS-CoV-2 infection at the immune defense-based protective phase while circumventing the inflammation-driven damaging phase.

Page $3 / 23$ 


\section{Results}

\section{Macrophage involved at the severe stage of COVID-19}

To better understand how macrophages impact COVID-19 progression, we compared immune cells and inflammatory factors in lung tissues obtained from autopsies of COVID-19 patients or healthy donors. First, histological changes in lung tissues from COVID-19 patients were examined. Compared to healthy lung tissues, this revealed extensive necrotizing bronchiolitis with necrotic bronchial epithelial cells and severe alveolitis with atrophy and desquamation, displayed in the lumen of the patient's lung (Figure 1A). Of note, pulmonary hemorrhagic infarct with abundant inflammatory infiltration (arrow heads) were extensively present through the whole alveoli and bronchial regions (Figure 1A). Recently, it was reported that proinflammatory $\mathrm{FCN}^{+}$monocyte-derived macrophages were mainly present and $\mathrm{FABP} 4^{+}$alveolar macrophages were greatly reduced in the bronchoalveolar lavage fluid from patients with severe COVID-19, whereas mild and moderate cases were characterized by the presence of highly clonally expanded CD $8^{+} \mathrm{T}$ cells $($ Liao et al., 2020). Therefore, we examined if macrophages were dominantly present in the diseased patient's lung. Immunostaining against pan macrophage marker CD68 showed abundant macrophages were extensively distributed through the whole lung tissue with aggregated phenotypes (Figure 1B), in agreement with the above-cited report. However, macrophages are multifaceted and distinct functions of macrophages highly depends on polarization, characterized generally as M1/pro-inflammatory or M2/anti-inflammatory macrophages. We thus further examined M1 macrophage marker CD80, and M2 macrophage marker CD163 (Figures 1C-F). The results revealed that cells positive for either $\mathrm{CD} 80$ or CD163 were both aberrantly represented in the patient's lung tissue (Figures 1CF). Indeed, $\mathrm{CD}_{68}{ }^{+}, \mathrm{CD} 80^{+}$and $\mathrm{CD} 163^{+}$macrophage populations were significantly expanded in the patient's lung tissue, suggesting expansion of both M1 and M2 macrophage populations in severe disease. We also examined several cytokines that are mainly produced by macrophages and found key pro-inflammatory cytokine IL -6 was intensively expressed in the lumen of the patient's lung tissue (Figure 1G). Taken together, the data supports a need to further examine the roles of M1 and M2 macrophages in COVID19 progression.

\section{Co-culture of lung cells and macrophages derived from hPSCs}

To further investigate the interaction among macrophages, lung cells and SARS-CoV-2, we established a co-culture model using cells derived from the same hPSC line (RUES2 or H1), which provide a genetically defined background for immune study. Several effective methods have recently been described (Dye et al., 2015; Hurley et al., 2020; Mou et al., 2012) for generating major cell types found in human lung tissues by directed differentiation of hPSCs using growth factors and chemicals to alter cell fate determining signaling pathways. Based on a protocol modified from ones previously developed by our lab and others (Chen et al., 2019; Huang et al., 2015; Huang et al., 2014), hPSCs were differentiated in a stepwise approach into definitive endoderm (DE), followed by anterior foregut endoderm (AFE), lung progenitor cells (LPs), and finally by specification to bronchial and alveolar lineage cells (Figures S1A-D, S2A$B)$. Single cell transcriptomic profiling identified in the cultures many expected populations that comprised 6 main clusters, including alveolar type II (AT2) cells (SP-B ${ }^{+}, \mathrm{SP}-\mathrm{C}^{+}, \mathrm{SP}-\mathrm{D}^{+}, \mathrm{ABCA3^{+ }}$ ), alveolar type I (AT1) cells $\left(\mathrm{AGER}^{+} \mathrm{AQP} 5^{+}\right)$, ciliated cells $\left(\mathrm{FOXJ1}^{+} \mathrm{CAPS}{ }^{+}\right.$), stromal cells $\left(\mathrm{DCN}^{+}\right)$, club cells $\left(\mathrm{SCGB} 3 \mathrm{~A} 1^{+} \mathrm{SCGB} 3 \mathrm{~A} 2^{+}\right)$, as well as low numbers of goblet cells $\left(\mathrm{MUC5} \mathrm{AC}^{+} \mathrm{MUC5} \mathrm{B}^{+}\right)$, basal progenitor cells $\left(\mathrm{P}_{63}{ }^{+}\right)$, and neuroendocrine cells $\left(\mathrm{ASCL} 1^{+} \mathrm{CALCA}{ }^{+}\right.$) (Figures S3A-D). ACE2, the putative SARS-CoV-2 receptor, and TMPRSS2, the co-effector for viral entry (Hoffmann, 2020), were detected in AT2, AT1 and ciliated cells, in clusters 0, 2, 3 (Figures S3E-F). The immunostaining results further validated that ACE2 is mainly co-expressed with SP-B or pro-SP-C in AT2 cells, and FOXJ1 in ciliated cells (Figure S2B), consistent with results previously reported in primary human lung tissues(Ziegler, 2020).

To generate macrophages and monocytes from hPSCs we used protocols published by us and others (Buchrieser et al., 2017; Cao et al., 2019; Duan et al., 2018; Lachmann et al., 2015; Lang et al., 2018). In brief, the hPSCs were first induced to mesoderm and then to vascular mesoderm cells, which were further differentiated to hematopoietic stem and progenitor cells (HSPCs). The HSPCs were induced to differentiate into functional macrophages by treatment with monocyte cytokines IL3 and Macrophage-colony stimulating factor (M-CSF) (Figures S4A-C). The hPSC-derived macrophages expressed major macrophage/monocyte markers such as CD14, CD11b and CD68 (Figure S4D) and were readily polarized to $C D 68^{+} \mathrm{CD} 206^{+}$macrophages, or CD68 ${ }^{+} \mathrm{FCN} 1^{+} \mathrm{STAT} 1^{\mathrm{hi}}$ macrophages (Figures 2A, D-H, Figure S5B) upon stimulation by IL-4 or IFNy and lipopolysaccharide (LPS) respectively (Figure S5A). Very few iM $\varphi$ s expressed ACE2 and TMPRSS2 based on single cell RNA (scRNA) profiling (Figure S5B). 
Next, the hPSC-induced lung cells (iLung) and macrophages (iM $\varphi$ ) were plated and cultured together in a 1:1 ratio (Figure 2A), similar to the ratio of lung cells and macrophages in distal bronchial or alveolar regions in human lung (Kyle J. Travaglini, 2020). The iLung was derived from the hPSC lines carrying a Doxycycline-inducible GFP reporter gene, which allowed the distinction of iLung and iM $\varphi$ in live cultures (Figure 2B). A significantly lower number of GFP+ iLung were observed after four-day co-culture with iM $\varphi$ of $M 1$ phenotype (iM1 $\varphi$ ), than seen in the co-culture with iM $\varphi$ of M2 phenotype (iM2 $\varphi$ ) or control 293T cells (Figure 2C). The scRNA profiles further revealed decreased expression of proliferation-associated genes MKI67 and TOP2A and increased expression of apoptosisrelated genes TP53, CASP3, BAX, MCL1, in the iLung co-cultured with $\mathrm{iM} 1 \varphi$, but not in co-cultures with iM2 $\varphi$ (Figure S6D). These results were in alignment with the phenotype of pro-inflammatory activities of $\mathrm{iM} 1 \varphi$, as scRNA-seq data detected a set of proinflammatory factors, IL 1B, IL 18, STAT1, FCN1, CXCL9, CXCL10, CXCL11, CXCL16, CCL2 highly expressed in iM1 $\varphi$ (Figure 2F-G, S5BC). In contrast, iM2 $\varphi$ mainly expressed anti-inflammatory factors or immunoregulatory genes such as TGM2, APOE, A2M, CCL 13, CCL26 and TREM2 (Figure 2F-G, S5B-C). Gene Ontology (GO) enrichment analysis comparing iM1 $\varphi$ and iM2 $\varphi$ revealed overactivation of differential signaling pathways such as pro-inflammatory IFNy, type I IFN, and neutrophil activation in iM1 $\varphi$; antiinflammatory and tissue damage-repair process of RNA catabolic process, protein co-localization to endoplasmic reticulum in iM $2 \varphi$ (Figures S6B, C). Similar phenotypes were observed in the iLung co-cultured with THP-1, an established monocyte line, upon activation of M1 or M2 phenotype (Figure 2C). The results indicate that activation of M1-macrophage was sufficient to create a toxic environment for the iLung even in the absence of viral infection.

\section{Immune response of macrophages following SARS-CoV-2 infection}

To model the immune response of macrophages to SARS-CoV-2 infection on lung cells, virus was introduced to the co-culture system (Figure 3A). As a first step to measure effects of macrophages on viral entry into lung cells, we used a SARS-CoV-2 pseudoentry virus, in which the backbone of a VSV-G pseudo-typed $\triangle$ G-luciferase virus carries the SARS-CoV-2 spike protein incorporated in the surface of the viral particle (Nie et al., 2020; Whitt, 2010). High luciferase activity was readily detected in iLung 24 hours after viral infection at $\mathrm{MOI}=0.01$, but not in $\mathrm{iM} \varphi$ or 293T in the co-culture (293T cells were used as a co-culture control, based on our preliminary data and previous report that the permissiveness of 293T to SARS virus is low (Wenhui Li, 2003)) (Figure 3B), and immunostaining confirmed that the viral luciferase protein was co-localized with ACE2 ${ }^{+}$cells in the iLung cultures (Figure S7B). Since the luciferase gene was expressed after the virus entered host cells, the luciferase activity correlated to the amount of viral entry host cells. Luciferase activity was markedly decreased in the co-cultures of iLung with all three lines of macrophages, iM $\varphi$, THP-1 and U937; no significant difference was found between hPSC-derived $\mathrm{iM} 1 \varphi$ or $\mathrm{iM} 2 \varphi$, indicating they have the similar inhibitory effects on viral infection (Figure 3B, Figure S7A). The results were further validated by immunostaining study that substantial decrease of luciferase protein was detected in iLung cells co-cultured with $\mathrm{iM} \varphi$, compared to those co-cultured with 293T (Figure S7A). The potential of $\mathrm{iM} \varphi$ to inhibit viral replication and spreading was next studied by infection with a patient-derived SARS-CoV-2 virus in the co-cultures. After 24 hours incubation with the SARS-CoV-2 virus (USA-WA1/2020, MOI =0.01), a significant decrease of viral protein was observed in the co-culture of iLung and $\mathrm{iM} \varphi$, compared to the co-culture of iLung and 293T. Strikingly, most SARS-CoV-2 virus SARS-N protein was detected in the $\mathrm{M} 2-\mathrm{iM} \varphi$ when co-cultured with iLung, while in contrast, substantial levels of SARS-N protein was detected in iLung cells in the co-cultures using M1-iM $\varphi$ or 293T (Figure 3D). The findings suggest that phagocytosis activity of $\mathrm{M} 2$-iM $\varphi$ functioned as protection for iLung from viral infection.

Several approaches were taken to thoroughly examine the immune response following iM $\varphi$ on SARS-CoV -2 infection. First, a cohort of cytokines and inflammatory factors that are known to be important for innate or adaptive immune responses were profiled, in the culture medium 24 hours after infection with the SARS-CoV-2 pseudo virus. Increased levels of IFNy, IL-6, and IL-18 were found in the co-cultures of iLung with $\mathrm{M} 1$-iM $\varphi$, while these were decreased in the co-cultures of iLung with M2-iM $\varphi$ (Figure 3C). To further characterize at the transcriptomic level the response of iLung and $\mathrm{iM} \varphi$ following viral infection, scRNA-seq was performed on the cocultures with SARS-CoV-2 pseudo virus infection and the analysis revealed that a set of anti-inflammatory factors and anti-viral activity related genes, such as CCL26, CCL13, ISG15, IFITM2 and IFITM3, were clearly upregulated when cultures contained M2-iM $\varphi$ (Figure 4A and C, Figures S8A). In contrast, pro-inflammatory factors, such as $I L-6, S 100 A 8 / A 9, L Y Z$ and TLR4 were highly expressed when the cultures contained M1-iM $\varphi$. (Figure 4A, C, Figures S8A). Gene enrichment analysis comparing iM1 $\varphi$ and iM $2 \varphi$ revealed over-activation of differential signaling pathways such as neutrophil degranulation and antigen processing and presentation, regulation of T cell mediated cytotoxicity in $\mathrm{iM} 1 \varphi$; granulocyte chemotaxis, response to interferon-gamma as well as phagocytosis in iM2 $\varphi$ (Figures $4 \mathrm{E}$ and F). Moreover, IL10 signaling related genes such as IL1ORA, IL 10RB, STAT3, SOCS3, TIMP1 and IRS2 were

Page 5/23 
enriched in iM2 $\varphi$, suggestive of anti-inflammatory macrophages (Figure S8D). The above results demonstrate a differential immune response of $\mathrm{iM} 2 \varphi$ versus $\mathrm{iM} 1 \varphi$ upon viral entry into host cells, as $\mathrm{iM} 2 \varphi$ increased phagocytosis activity and released anti-

inflammatory factors, while $\mathrm{iM} 1 \varphi$ increased antigen-presenting activity and released pro-inflammatory factors.

Correlating with the above phenotypes, up-regulation of cell growth arrest or death-related genes, such as GAS6, BTG2, PDCD6, CCAR1, TP53/11, TP53INP1, and activation of programmed death signaling pathways as well as higher mitochondrial genes, MT-CYB,MT-CO1, MT-CO2, MT-ND1 (Figure S8B and C), were detected in the co-cultures with iLung with iM1 $\varphi$, but not with iM2 $\varphi$ (Figure S8B). Previous studies by us (Yuling Han, 2020) and others (Conti et al., 2020) suggested that lung cells display selfimmune defense after SARS-CoV-2 infection, releasing proinflammatory factors, such as CXCL2, CCL2, CXCL3 and IL 1A, as well as $B C R C 3, A A D A C$, and $A T P B 4$. The GO and KEGG analysis in our current co-culture based data suggest that upregulation in pathway networks including leukocyte chemotaxis NF-KB signaling, IL-17 signaling, viral protein interaction with cytokine-cytokine receptor, and response to type I interferon, combined with the pro-inflammatory reaction of M1 macrophages, could lead to further pulmonary inflammation and damage (Figure S8E). Moreover, the scRNA-seq profiling data further validated the immunostaining results showing that few if any iLung cells in the co-culture with $\mathrm{M} 2-\mathrm{iM} \varphi$ displayed detectable viral gene expression, in contrast to a significantly higher number of iLung cells in the co-culture with $\mathrm{M} 1-\mathrm{iM} \varphi$ (Figure 4B). Most infected AT2 cells and ciliated cells were also found in the co-culture with $\mathrm{M} 1-\mathrm{iM} \varphi$, indicating a stronger protective effect on iLung cells by $\mathrm{M} 2$-iM $\varphi$ (Figure 4D). Altogether, these findings suggest that activation of pro-inflammatory macrophages can aggravate lung cell damage, beyond the destruction by viral infection; in contrast, activation of anti-inflammatory macrophages provides a protective effect for lung cells from viral infection.

\section{Blockage of ACE2 enhances elimination of SARS-CoV-2 by macrophages}

Several studies (Tay et al., 2020) on mild or recovered COVID-19 cases indicated that in a healthy immune response, neutralizing antibodies produced in these individuals can block viral infection, followed by alveolar macrophages recognizing the neutralized viruses and clearing them by phagocytosis. We sought to model this process using an ACE2 blocking antibody to inhibit virus entry to target cells, thus decreasing the viral loads (Figure 5A), to test if this enhances phagocytosis activity of macrophages. As expected, incubation with ACE2 blocking antibody two hours prior to infection of SARS-CoV-2 pseudo virus, reduced markedly the luciferase activities in co-cultures of iLung with either $\mathrm{M} 1$ or $\mathrm{M} 2-\mathrm{iM} \varphi$, although the decrease of luciferase signal was most pronounced in the cocultures with $\mathrm{M} 2$-iM $\varphi$ (Figure 5B, Figure S9A). Immunostaining results validated that luciferase protein dramatically decreased in the iLung cells co-cultured with iM $\varphi$, compared to those co-cultured with 293T (Figure S9A).

Similarly, immunostaining results from the experiments performed using SARS-CoV-2 virus further revealed that that most SARS$\mathrm{CoV}-2-\mathrm{N}$ protein was found in the $\mathrm{M} 2-\mathrm{iM} \varphi$, but not in the iLung cells, while the $\mathrm{N}$ protein was clearly found in iLung cells in the coculture with $\mathrm{M} 1-\mathrm{iM} \varphi$ or 293T (Figure $5 \mathrm{C}$ ). These results demonstrated that an early intervention of viral infection by blocking ACE2 in target cells can increase the clearance of virus by macrophages, especially synergizing with the phagocytosis activity of M2macophages to further provide protection for target cells and reduce the damage by inflammatory factors produced by M1macrophages.

\section{Discussion}

The study of human host-immune systems with pathogens has depended historically on the use of animal models, largely due to limited cell resources derived from human tissues. Immune research on COVID-19 is limited by the types of models available for study. Recently, a transgenic mouse strain(McCray et al., 2007) has been made with human ACE2 expression regulated by human cytokeratin-18 promoter, but the ACE2 expression in human is more complex than that in the mice. Another model is ferret(Kim et al., 2020), which can be infected with SARS-CoV-2, but does not develop hyper-inflammation in the lung. Recent advances in stem cell biology, especially the technology to differentiate human pluripotent stem cells (hPSCs) into functional immune cell types, provide a rigorous human system for studying immunology and therapeutics. In this report, we describe a new cell co-culture system in which the immune cells, specifically monocytes/macrophages, and lung lineage cells are produced by directed differentiation of hPSCs. Several key features make the human cell model an ideal system for studying immunology of SARS-CoV-2. The model contains the host cells and immune cells from the same hPSC lines, avoiding concern of histocompatibility, while it can provide abundant numbers of cells with a genetically defined background for robust mechanistic or therapeutic studies. 
The innate immune response mainly mediated by macrophages or granulocytes, responding to tissue damage caused by SARSCoV-2 infection, likely contributes to acute respiratory distress syndrome (ARDS) that is characterized by the rapid onset of widespread inflammation in lung and subsequent respiratory failure (Xu et al., 2020). Our study in COVID-19 patient samples validated a correlation of macrophages and the disease, showing a heavy infiltration of pro-inflammatory macrophages in tissue samples from distal lung regions with high levels of inflammatory cytokine IL- 6 in the severe cases. The macrophage and lung cell co-culture model combined with single cell transcriptomics was then applied to interrogate the differential immune responses of proor anti-inflammatory macrophages following SARS-CoV-2 infection. We discovered that pro- and anti-inflammatory macrophages both have similar capacity to eliminate the virus in the context of a moderate viral load. However, the immune reaction of proinflammatory macrophages following SARS-CoV-2 pseudo-virus infection led to more damage on lung cells and secretion of a set of inflammatory factors including IL6, IL18 and CXCL10 that are known to be mediators in dysfunctional immune responses and cytokine release syndrome (CRS). In contrast, anti-inflammatory macrophages protected lung cells from viral infection, and diminished pulmonary inflammation by phagocytosis and production of anti-inflammatory factors related in IL10 signaling. Finally, inhibiting viral entry in target cells using an ACE2 blocking antibody, diminished viral infection and enhanced the elimination of viruses. In particular, the intervention on viral entry can synergize with the phagocytosis and antiviral activity of macrophages, resulting in a more pronounced clearance of virus and protection of target cells.

The immune responses induced by SARS-CoV-2 infection can be clinically divided into two phases, the first immune defense-based protective phase and the second inflammation-driven damaging phase (Shi et al., 2020; Tay et al., 2020). Boosting immune responses in the first phase of the viral incubation while suppressing it in the second phase, could help eliminate the virus and preclude disease progression to severe stages. Our study demonstrates the potential and significance of anti-inflammatory macrophages for inhibiting viral infection and protecting host cells. Furthermore, several ACE2 blocking antibodies, or virus neutralizing antibodies (Long Chen, 2020) have been reported as currently under development or testing in clinical trials to treat COVID-19 patients (https://clinicaltrials.gov/ct2/show/NCT04335136). On the other hand, cell-based immunotherapy using autologous or stem cell-derived macrophage has been studied or tested in clinical trials (https://doi.org/10.1186/ISRCTN10368050) to treat a variety of human diseases, especially the application of anti-inflammatory macrophages in treating chronic inflammatory diseases(Moroni et al., 2019) (Chan and Viswanathan, 2019; Rodell et al., 2019). With these resources and information on hand, the strategy we developed in this study, combining blocking ACE2 receptor while boosting anti-inflammatory macrophages, provides some new ideas that can be employed to help combat this deadly contagious disease.

\section{Materials And Methods}

\section{STAR * METHODS}

Detailed methods are provided in the outline version of this paper and included the following:

\section{KEY RESOURCES TABLE}




\begin{tabular}{|c|c|c|}
\hline $\begin{array}{l}\text { REAGENT or } \\
\text { RESOURCE }\end{array}$ & SOURCE & IDENTIFIER \\
\hline \multicolumn{3}{|l|}{ Antibodies } \\
\hline $\begin{array}{l}\text { Mouse monoclonal } \\
\text { anti-CD } 68\end{array}$ & eBioscience & \#14-0688-82 \\
\hline $\begin{array}{l}\text { Rabbit polyclonal } \\
\text { anti-CD } 80\end{array}$ & BOSTER & \#A00196-1 \\
\hline CD80-PE, human & miltenyi Biotec & \#130-117-683 \\
\hline CD206-PE, human & miltenyi Biotec & \#130-095-220 \\
\hline $\begin{array}{l}\text { Rabbit monoclonal } \\
\text { anti-CD163 }\end{array}$ & Abcam & \#ab182422 \\
\hline $\begin{array}{l}\text { Rabbit polyclonal } \\
\text { anti-IL6 }\end{array}$ & Affinity & \#DF6087 \\
\hline $\begin{array}{l}\text { Goat polyclonal } \\
\text { anti-ACE2 }\end{array}$ & R\&D system & \#AF933 \\
\hline $\begin{array}{l}\text { PE-conjugated } \\
\text { CD43 }\end{array}$ & eBioscience & \#еBio84-3C1 \\
\hline $\begin{array}{l}\text { APC-conjugated } \\
\text { CD34 }\end{array}$ & BD Biosciences & clone 581 \\
\hline $\begin{array}{l}\text { PE-conjugated } \\
\text { CD68 }\end{array}$ & Biolegend & clone Y1/82A \\
\hline $\begin{array}{l}\text { APC-conjugated } \\
\text { CD11b }\end{array}$ & Biolegend & clone ICRF44 \\
\hline $\begin{array}{l}\text { FITC-conjugated } \\
\text { CD14 }\end{array}$ & Biolegend & clone HCD14 \\
\hline $\begin{array}{l}\text { Anti-NKX2.1 } \\
\text { Antibody }\end{array}$ & Seven Hills Bioreagents & \#WRAB-1231 \\
\hline $\begin{array}{l}\text { Anti-FOXA2 } \\
\text { Antibody }\end{array}$ & Santa Cruz & \#sc-6554 \\
\hline Anti-SOX2 Antibody & Santa Cruz & \#sc-17320 \\
\hline Anti-SP-B Antibody & Seven Hills Bioreagents & \#WRAB-48604 \\
\hline $\begin{array}{l}\text { Anti-Pro-SP-C } \\
\text { Antibody }\end{array}$ & Seven Hills Bioreagents & \#WRAB-9337 \\
\hline $\begin{array}{l}\text { Anti-FOXJ1 } \\
\text { Antibody }\end{array}$ & Sigma-Aldrich & \#HPA005714-1 \\
\hline $\begin{array}{l}\text { Firefly luciferase } \\
\text { Monoclonal } \\
\text { Antibody (CS 17) }\end{array}$ & Thermo Fisher Scientific & \#35-6700 \\
\hline $\begin{array}{l}\text { Recombinant Anti- } \\
\text { Firefly Luciferase } \\
\text { antibody }\end{array}$ & Abcam & \#ab185924 \\
\hline $\begin{array}{l}\text { Mouse Anti-SARS- } \\
\text { CoV-Spike antibody }\end{array}$ & Provided by Dr. Tom Moran & 2B3E5 \\
\hline $\begin{array}{l}\text { Donkey anti-Mouse } \\
\text { IgG }(\mathrm{H}+\mathrm{L}) \text { Highly } \\
\text { Cross-Adsorbed } \\
\text { Secondary } \\
\text { Antibody, Alexa } \\
\text { Fluor } 488\end{array}$ & Thermo Fisher Scientific & \#A-21202 \\
\hline
\end{tabular}


Alexa Fluor 488

Jackson Immunoresearch Labs

AffiniPure Donkey

Anti-Guinea Pig lgG

$(\mathrm{H}+\mathrm{L})$

Donkey anti-Mouse Thermo Fisher Scientific

IgG $(\mathrm{H}+\mathrm{L})$ Highly

Cross-Adsorbed

Secondary

Antibody, Alexa

Fluor 594

Donkey anti-Rabbit Thermo Fisher Scientific

$\lg \mathrm{G}(\mathrm{H}+\mathrm{L})$

Secondary

Antibody, Alexa

Fluor 594

conjugate

Donkey anti-Rabbit Thermo Fisher Scientific

$\operatorname{lgG}(\mathrm{H}+\mathrm{L})$

Secondary

Antibody, Alexa

Fluor 647

conjugate

Donkey anti-Mouse Thermo Fisher Scientific

$\lg G(\mathrm{H}+\mathrm{L})$

Secondary

Antibody, Alexa

Fluor 647

Donkey anti-Goat

IgG $(\mathrm{H}+\mathrm{L})$ Cross-

Thermo Fisher Scientific

\#A-21447

Adsorbed

Secondary

Antibody, Alexa

Fluor 647

Donkey anti-

Chicken lgG $(\mathrm{H}+\mathrm{L})$

Cross-Adsorbed

Secondary

Antibody, Alexa

Fluor 488

Donkey anti-Sheep

IgG (H+L) Cross-

Adsorbed

Secondary

Antibody, Alexa

Fluor 647

\section{Chemicals,}

Peptides, and

Recombinant

Proteins

\begin{tabular}{|llc|}
\hline Activin A & R\&D Systems & \#338-AC-500/CF \\
\hline Y-27632 & MedchemExpress & \#HY-10583 \\
$\begin{array}{l}\text { Recombinant } \\
\text { Human BMP-4 } \\
\text { Protein }\end{array}$ & R \& D Systems & \#314-BP \\
$\begin{array}{l}\text { Recombinant } \\
\text { Human bFGF }\end{array}$ & R\&D Systems & \#233-FB-500 \\
$\begin{array}{l}\text { Dorsomorphin } \\
\text { dihydrochloride }\end{array}$ & R\&D Systems & \#3093/50 \\
\hline SB431542 & R\&D Systems & $\# 1614 / 50$ \\
\hline IWP2 & R\&D Systems & $\# 3533 / 50$
\end{tabular}

Page 9/23

\#A-21203

\#A-21207

\#A-31573

\#A-31571

Jackson Immunoresearch Labs \#703-545-155

\#A-21448

Thermo Fisher Scientific

\#A-21448 


\begin{tabular}{|c|c|c|}
\hline CHIR99021 & Cayman Chemical & \#13122 \\
\hline $\begin{array}{l}\text { Recombinant } \\
\text { Human FGF-10 } \\
\text { Protein }\end{array}$ & R\&D Systems & \#345-FG-250 \\
\hline $\begin{array}{l}\text { Recombinant } \\
\text { Human KGF/FGF-7 } \\
\text { Protein }\end{array}$ & R\&D Systems & \#251-KG-01M \\
\hline Retinoic acid & Sigma-Aldrich & \#R2625 \\
\hline Dexamethasone & Sigma-Aldrich & \#D4902 \\
\hline 8-Bromo-cAMP & Sigma-Aldrich & \#B5386 \\
\hline IBMX & Sigma-Aldrich & \#15879 \\
\hline $\begin{array}{l}\text { Recombinant } \\
\text { Human VEGF } \\
\text { Protein }\end{array}$ & R\&D Systems & \#293-VE-500/CF \\
\hline $\begin{array}{l}\text { Recombinant } \\
\text { Human IL-3 Protein }\end{array}$ & R\&D Systems & \#203-IL-050/CF \\
\hline $\begin{array}{l}\text { Recombinant } \\
\text { Human M-CSF } \\
\text { Protein }\end{array}$ & R\&D Systems & \#216-MC-025 \\
\hline $\begin{array}{l}\text { Recombinant } \\
\text { Human IL4 Protein }\end{array}$ & R\&D Systems & \#204-IL-050 \\
\hline IFNy & R\&D Systems & \#285-IF-100 \\
\hline LPS & Sigma-Aldrich & \#L4391-1MG \\
\hline DAPI & Santa Cruz & \#sc-3598 \\
\hline Hoechst 33342 & Sigma-Aldrich & \# B2261-100mg \\
\hline $\begin{array}{l}\text { Wright-Giemsa } \\
\text { Stain }\end{array}$ & Sigma-Aldrich & \#WG16-500ML \\
\hline \multicolumn{3}{|l|}{ Culture Medium } \\
\hline F12 & Gibco Thermo Fisher & \#31765035 \\
\hline$\beta$-mercaptoethanol & Sigma Aldrich & \#M3148 \\
\hline $\begin{array}{l}\text { Penicillin- } \\
\text { Streptomycin } \\
(5,000 \mathrm{U} / \mathrm{mL})\end{array}$ & Gibco Thermo Fisher & \#15070063 \\
\hline $\begin{array}{l}\text { MEM Non-Essential } \\
\text { Amino Acids } \\
\text { Solution (100X) }\end{array}$ & Gibco Thermo Fisher & $\# 11140050$ \\
\hline IMDM & Gibco Thermo Fisher & \#21056023 \\
\hline $\begin{array}{l}\text { GlutaMAX } \\
\text { Supplement }\end{array}$ & Thermo Fisher Scientific & \#35050079 \\
\hline Accutase & Stemcell Technologies & \#07920 \\
\hline Matrigel & Corning & \#354234 \\
\hline Fibronectin (FN) & Thermo Fisher Scientific & \#356008 \\
\hline N2 supplement & Thermo Fisher Scientific & \#17502-048 \\
\hline B27 & Thermo Fisher Scientific & \#12587-010 \\
\hline
\end{tabular}




\begin{tabular}{|c|c|c|}
\hline DMEM/F12 & Thermo Fisher Scientific & \#10565-018 \\
\hline $\begin{array}{l}\text { Knockout serum } \\
\text { replacement(KOSR) }\end{array}$ & Thermo Fisher Scientific & \#10828-028 \\
\hline FBS & Gibco Thermo Fisher & \#10099141C \\
\hline Monothioglycerol & Sigma Aldrich & \#M6145 \\
\hline Ascorbic acid & Sigma Aldrich & \#A4403 \\
\hline $\begin{array}{l}\text { Bovine serum } \\
\text { albumin(BSA) }\end{array}$ & Sigma Aldrich & \#A9418 \\
\hline $\begin{array}{l}\text { Experimental } \\
\text { Models: Cell Lines }\end{array}$ & Seven Hills Bioreagents & \#WRAB-1231 \\
\hline hESC line H1 & Harvard University & \#0014 \\
\hline hESC line-RUES2 & The Rockefeller University & \#0013 \\
\hline HEK293T & ATCC & \#CRL-11268 \\
\hline Vero E6 & ATCC & \#CRL-1586 \\
\hline THP-1 & ATCC & \#TIB-202 \\
\hline U937 & ATCC & \#CRL-1593.2 \\
\hline $\begin{array}{l}\text { Mouse embryonic } \\
\text { fibroblasts }\end{array}$ & Global Stem & \#GSC-6001G \\
\hline \multicolumn{3}{|c|}{ Software and Algorithms } \\
\hline Cell Ranger & 10X Genomics & $\begin{array}{l}\text { https://support.10xgenomics.com/single-cell-gene- } \\
\text { expression/software/overview/welcome }\end{array}$ \\
\hline Scran & $\begin{array}{l}\text { Lun ATL, McCarthy DJ, Marioni JC } \\
\text { (2016). "A step-by-step workflow for } \\
\text { low-level analysis of single-cell RNA- } \\
\text { seq data with } \\
\text { Bioconductor." F1000Res., 5, } 2122 \text {. } \\
\text { doi: 10.12688/f1000research.9501.2. }\end{array}$ & https://bioconductor.org/packages/release/bioc/html/scran.html \\
\hline Rstudio & Rstudio & https://rstudio.com \\
\hline $\begin{array}{l}\text { Seurat R package } \\
\text { v3.1.4 }\end{array}$ & (Butler et al., 2018) & https://satijalab.org/seurat/ \\
\hline DAVID6.8 & LHRI & https://david.ncifcrf.gov/home.jsp \\
\hline $\begin{array}{l}\text { Adobe illustrator } \\
\text { CC2017 }\end{array}$ & Adobe & https://www.adobe.com/product/photoshop.html \\
\hline $\begin{array}{l}\text { Graphpad Prism } \\
8.0\end{array}$ & Graphpad software & https://www.graphpad.com \\
\hline LEGENDplex v8.0 & Biolegend & https://www.biolegend.com/en-us/legendplex \\
\hline FlowJo v x.0.7 & BD Biosciences & https://www.flowjo.com/ \\
\hline ToppCell Atlas & Toppgene & https://toppgene.cchmc.org/ \\
\hline
\end{tabular}

\section{METHOD DETAILS}


The paraffin-embedded lung tissues were acquired from the department of pathology in the $3^{\text {rd }}$ people's hospital of Shenzhen, China. They recently reported the pathological changes of lungs from a 66-year-old male died in critical COVID-19 infection(Weiren Luo). The patient developed respiratory failure and septic shock during the treatment and was done with transplant. Informed consent was obtained from the patient and family. The diagnosis of COVID-19 pneumonia was based on the "Coronavirus Pneumonia Prevention and Control Plan" (7th edition) newly issued by the National Health Commission, China (Commission, 2020). Nasopharyngeal swabs were collected and COVID-19 was detected by real-time polymerase chain reaction. Infection was defined as at least two positive test results. Surgical informed consent was obtained and the study was approved by IRB in the third People's hospital of Shenzhen.

\section{hPSC lung differentiation}

Protocols for maintenance of hPSCs and generation of lung cells were slightly modified from previous studies (Chen et al., 2019; Huang et al., 2014). The hESC line-RUES2 or H1 was cultured on irradiated mouse embryonic fibroblasts (Global Stem, cat. no. GSC$6001 \mathrm{G}$ ) at a density of 20,000-25,000 cells/cm² in a medium of DMEM/F12, 20\% knockout serum replacement (Life Technologies), $0.1 \mathrm{mM} \beta$-mercaptoethanol (Sigma Aldrich) and $20 \mathrm{ng} / \mathrm{ml} \mathrm{bFGF} \mathrm{(R \& D} \mathrm{Systems),} \mathrm{and} \mathrm{medium} \mathrm{was} \mathrm{changed} \mathrm{daily.} \mathrm{hESC} \mathrm{cultures} \mathrm{were}$ maintained in an undifferentiated state at $37^{\circ} \mathrm{C}$ in a $5 \% \mathrm{CO} 2 /$ air environment until stem cells reached about $90 \%$ confluence.

hESC differentiation into endoderm was performed in serum-free differentiation (SFD) medium of IMDM/F12 (3:1) (Life Technologies) supplemented with N2 (Life Technologies), B27 (Life Technologies), $50 \mu \mathrm{g} / \mathrm{ml}$ ascorbic acid, $2 \mathrm{mM}$ Glutamax, $0.4 \mu \mathrm{M}$ monothioglycerol, $0.05 \% \mathrm{BSA}$ at $37{ }^{\circ} \mathrm{C}$ in a $5 \% \mathrm{CO}_{2} / 5 \% \mathrm{O}_{2} / 95 \% \mathrm{~N}_{2}$ environment. hESCs were treated with Accutase and plated onto low attachment 6-well plates (Corning Incorporated, Tewksbury MA), resuspended in endoderm induction medium containing $10 \mu \mathrm{M}$ Y-27632, $0.5 \mathrm{ng} / \mathrm{ml}$ human BMP-4, $2.5 \mathrm{ng} / \mathrm{ml}$ human bFGF, $100 \mathrm{ng} / \mathrm{ml}$ human Activin A, for 72-76 hours dependent on the formation rates of endoderm cells. On day 3 , the endoderm bodies were dissociated into single cells using $0.05 \%$ Trypsin/0.02\% EDTA and plated onto fibronectin-coated, 24-well tissue culture plates ( 100,000-150,000 cells/well). For induction of anterior foregut endoderm (AFE), the endoderm cells were cultured in SFD medium supplemented with $1.5 \mu \mathrm{M}$ Dorsomorphin dihydrochloride (R\&D Systems) and $10 \mu \mathrm{M}$ SB431542 (R\&D Systems) for $48 \mathrm{~h}$, and then switched to $24 \mathrm{~h}$ of $10 \mu \mathrm{M}$ SB431542 and $1 \mu \mathrm{M}$ IWP2 (R\&D Systems) treatment. For induction of early stage lung progenitor cells (day 6-15), the resulting anterior foregut endoderm was treated with $3 \mu \mathrm{M}$ CHIR99021, $10 \mathrm{ng} / \mathrm{ml}$ human FGF10, $10 \mathrm{ng} / \mathrm{ml}$ human FGF-7, $10 \mathrm{ng} / \mathrm{ml}$ human BMP-4 and 50-60nM all-trans retinoic acid (ATRA), in SFD medium for 8-10 d. The day 10-15 cultures were maintained in a $5 \% \mathrm{CO}_{2} /$ air environment. On days 15 and 16 , the lung progenitor cells were replated after one minute trypsinization onto fibronectin-coated plates, in the presence of SFD containing $3 \mu \mathrm{M}$ CHIR99021, $10 \mathrm{ng} / \mathrm{ml}$ human FGF10, $10 \mathrm{ng} / \mathrm{ml}$ human FGF7, in a $5 \% \mathrm{CO}_{2} /$ air environment. For differentiation of mature lung cells (day 25 to 55), cultures were re-plated after brief trypsinization onto 3.3\% Matrigel-coated 24-well plates in SFD media containing maturation components containing $3 \mu \mathrm{M}$ CHIR99021, $10 \mathrm{ng} / \mathrm{ml}$ human FGF-10; $10 \mathrm{ng} / \mathrm{ml}$ human FGF-7, and DCl (50 nM Dexamethasone, 0.1 mM 8-bromo-cAMP (Sigma Aldrich ) and 0.1 mM IBMX (3,7-dihydro-1-methyl-3-(2-methylpropyl)-1Hpurine-2,6-dione) (Sigma Aldrich)). The protocol details are summarized in Figure S1A.

All embryonic stem cell studies were approved by the Institutional Review Board (IRB) at the University of Chicago, or by the TriInstitutional ESCRO committee (Weill Cornell Medicine, Memorial Sloan Kettering Cancer Center, and Rockefeller University).

\section{hPSC macrophage differentiation}

We derived macrophages from hESC line H1 or RUES2 and adapted based on previously reported protocols(Cao et al., 2019; Duan et al., 2018; Lachmann et al., 2015). For macrophage differentiation, at day -2, hESCs were digested into single-cell suspension by 1 $\mathrm{mg} / \mathrm{ml}$ Accutase (Stemcell Technologies) and plated onto Matrigel-coated culture dishes at a density of $2 \times 10^{4}$ cells $/ \mathrm{cm}^{2}$ in mTeSR 1 medium with 5uM Y27632 (MedchemExpress). After $24 \mathrm{~h}, \mathrm{Y} 27632$ was withdrawn from the medium and cells were cultured for another $24 \mathrm{~h}$. At day 0, cells were firstly induced by macrophage differentiation basal medium (SFD-M) which is RPMI 1640 medium supplemented with 2\% B27 (Thermo Fisher Scientific), 1\% L-GlutaMAX-I and $50 \mu \mathrm{g} / \mathrm{ml}$ ascorbic acid (Sigma Aldrich) and $10 \mathrm{ng} / \mathrm{ml}$ BMP4 (R\&D Systems) for $24 \mathrm{~h}$. Afterward, the medium was changed to SFD-M medium containing $10 \mathrm{ng} / \mathrm{ml} \mathrm{BMP4} \mathrm{and} 2 \mu \mathrm{M}$ GSK3 inhibitor CHIR99021 (Cayman Chemical) for another $48 \mathrm{~h}$. At day 3, cells were replated onto Matrigel-coated dishes at a density of $4 \times$ $10^{4}$ cells/ $\mathrm{cm}^{2}$ in SFD-M medium with $50 \mathrm{ng} / \mathrm{ml} \mathrm{VEGF} \mathrm{(R \& D} \mathrm{Systems)} \mathrm{and} 10 \mathrm{ng} \mathrm{ng} / \mathrm{ml}$ FGF2 (R\&D Systems) for $48 \mathrm{~h}$. At day 5, the medium was replaced with basal medium with $50 \mathrm{ng} / \mathrm{ml} \mathrm{VEGF,} 10 \mathrm{ng} \mathrm{ng} / \mathrm{ml} \mathrm{FGF2}$ and 10uM TGF $\beta$ inhibitor SB431542 (R\&D Systems) for another $72 \mathrm{~h}$. At day 8-10, floating cells were collected and medium was changed and supplemented with $50 \mathrm{ng} / \mathrm{ml} \mathrm{M}$ CSF and 10ng/ml IL3 (R\&D Systems) for another 3-5 days. From day 11-13 onward, the medium was changed to SFD-M medium

Page $12 / 23$ 
with $50 \mathrm{ng} / \mathrm{ml} \mathrm{M}$-CSF for 3 days. All differentiation steps were cultured under normoxic conditions at $37{ }^{\circ} \mathrm{C}, 5 \% \mathrm{CO}_{2}$. The protocol details are summarized in Figure S4A.

All embryonic stem cell studies were approved by the Institutional Review Board (IRB) at the University of Chicago, or by the TriInstitutional ESCRO committee (Weill Cornell Medicine, Memorial Sloan Kettering Cancer Center, and Rockefeller University).

\section{hPSC monocyte polarization}

hPSC-derived $\mathrm{CD}_{14}{ }^{+}$cells were plated on tissue culture plates at a density of $2 \times 10^{4}$ cells $/ \mathrm{cm}^{2}$ in SFD-M medium supplemented with $50 \mathrm{ng} / \mathrm{mL}$ M-CSF. After 2 days of culture, monocytes differentiated into M0 macrophages and polarized to M1 or M2 macrophages. For macrophages polarization, 100ng/mL LPS (Sigma-Aldrich) and 10ng/mL IFNY (R\&D Systems) were added for M1 induction, or $20 \mathrm{ng} / \mathrm{m} \mathrm{IL}-4$ (R\&D Systems) was added for M2 induction in SFD-M medium supplemented with $50 \mathrm{ng} / \mathrm{mL}$ M-CSF, respectively. These cells were cultured for another three days before examination for expression of the M1 or M2 makers.

\section{Giemsa Staining}

Differentiating day 11-13 monocytes/macrophages were fixed on slides using Cytospin, followed by staining using Wright-Giemsa Stain (Sigma-Aldrich) according to the manufacturer's instructions.

\section{Immunohistochemical staining}

Histological study of lung tissues was performed on paraffin-embedded sections as previously described (Li et al., 2014). For immunohistochemical staining, paraffin-embedded sections were deparaffinized and incubated with primary antibodies at $4^{\circ} \mathrm{C}$ overnight and secondary antibodies at room temperature for $1 \mathrm{~h}$. Primary antibodies and secondary antibodies are described in the supplementary Table. Nuclei were counterstained by Hoechst 33342 (Sigma). positive cells in lungs were randomly counted from different visions of slides by confocal microscopy. 12 views in each lung section were counted and averaged cell numbers per $0.04^{2}$ $\mathrm{mm}$ were used to define the distributions of positive cells in the lung tissues as described (Plasschaert et al., 2018). Living cells in culture were directly fixed in $4 \%$ paraformaldehyde for $25 \mathrm{~min}$, followed with 15 min permeabilization in $1 \%$ triton X-100. For immunofluorescence, cells or tissue sections were immunostained with antibodies and counterstained with 4,6-diamidino-2phenylindole (DAPI). Adjacent sections stained with $\mathrm{H}$ and $\mathrm{E}$ were used for comparison. The antibodies used for immunostaining or western blot experiments are listed in the key resource table.

\section{Fluorescent activated cell sorting (FACS)}

For FACS analysis, cells were resuspended in a FACS buffer (PBS with $0.1 \%$ BSA and $2.5 \mathrm{mM}$ EDTA). The cell suspension was then stained with PE-conjugated CD43 (Biolegend, clone MEM-59), APC-conjugated CD34 (BD, clone 581) to detect hematopoietic stem/progenitor cells (HSPC). PE-conjugated CD68 (Biolegend, clone Y1/82A), APC-conjugated CD11b (Biolegend, clone ICRF44), FITC-conjugated CD14 (Biolegend, clone HCD14) were used to detect monocyte/macrophages. Basically, cells were incubated with antibodies for 30 minutes at $4^{\circ} \mathrm{C}$, followed with washed and suspended in $0.1 \%$ BSA/PBS buffer. PE and APC filters were then used to detect cells double positive for CD43 and CD34 or CD68 and CD11b by signal intensity gating, FITC and APC were used to detect cells double positive for CD14 and CD11b. Negative controls stained with control IgG instead of primary antibodies were always performed with sample measurements. Flowcytometry machine of BD FACSAria II and software of Flowjo were mainly used to collect and analyze the flowcytometry data.

\section{Cytokine analysis}

Cytokines including hIL-1ß, IFN- $\alpha 2$, hIFN- $y$, hTNF-a, hMCP-1, hIL-6, hIL-8, hIL-10, hIL-12p70, IL-17A, hIL-18, hIL-23, hIL-33 were detected according to the instruction of LEGENDplex ${ }^{\mathrm{TM}}$ kit (Biolegend, cat. no. 740808). In brief, 25ul supernatant was taken from the coculture medium and mixed with $25 \mu \mathrm{l}$ of premixed beads and $25 \mu \mathrm{l}$ of detection antibodies. The mixtures were placed on a shaker at 400 r.p.m. for $2 \mathrm{~h}$ at RT. Then $25 \mu$ l of SA-PE was added to each tube and placed on a shaker at 500 r.p.m. for 30 min. The data were obtained by flow cytometry (FACSAria II, BD) and were analyzed with LEGENDplex v.8.0 (Biolegend).

\section{SARS-CoV-2-Pseudo-Entry Viruses}


Recombinant Indiana VSV (rVSV) expressing SARS-CoV-2 spikes was generated as previously described (Nie et al., 2020; Whitt, 2010; Zhao et al., 2017). HEK293T cells were grown to $80 \%$ confluency before transfection with pCMV3-SARS-CoV2-spike (kindly provided by Dr. Peihui Wang, Shandong University, China) using FuGENE 6 (Promega). Cells were cultured overnight at $37^{\circ} \mathrm{C}$ with $5 \% \mathrm{CO}_{2}$. The next day, the media was removed and VSV-G pseudotyped $\Delta$ G-luciferase (G* $\Delta G$-luciferase, Kerafast) was used to infect the cells in DMEM at an MOI of 3 for $1 \mathrm{hr}$ before washing the cells with 1X DPBS three times. DMEM supplemented with $2 \%$ FBS and 100 I.U. $/ \mathrm{mL}$ penicillin and $100 \mu \mathrm{g} / \mathrm{mL}$ streptomycin was added to the infected cells and they were cultured overnight as described above. The next day, the supernatant was harvested and clarified by centrifugation at $300 \mathrm{xg}$ for $10 \mathrm{~min}$ before aliquoting and storing at $-80^{\circ} \mathrm{C}$.

\section{SARS-CoV-2 infections}

SARS-CoV-2, isolate USA-WA1/2020 (NR-52281) was deposited by the Center for Disease Control and Prevention and obtained through BEI Resources, NIAID, NIH. SARS-CoV-2 was propagated in Vero E6 cells in DMEM supplemented with 2\% FBS, 4.5 g/L Dglucose, $4 \mathrm{mM}$ L-glutamine, $10 \mathrm{mM}$ Non-Essential Amino Acids, $1 \mathrm{mM}$ Sodium Pyruvate and $10 \mathrm{mM}$ HEPES as described previously(Blanco-Melo et al., 2020). hESC-derived lung and macrophage co-cultures in 96-well plates were infected with SARS-CoV-2 for $24 \mathrm{~h}$ at an $\mathrm{MOI}$ of 0.01 in the medium containing SFD:SFD-M=1:1. For immunofluorescence staining, cells were washed three times in PBS and fixed for $24 \mathrm{~h}$ in $5 \%$ formaldehyde for $24 \mathrm{~h}$ for immunofluorescent staining, prior to safe removal from the BSL-3 facility.

All work involving live SARS-CoV-2 was performed in the CDC/USDA-approved BSL-3 facility of the Global Health and Emerging Pathogens Institute at the Icahn School of Medicine at Mount Sinai in accordance with institutional biosafety requirements

\section{SARS-CoV-2 entry virus infections.}

To assay pseudo-typed virus infection, cells were seeded in 96 well plates. Pseudo-typed virus was added for MOI=0.01. At $2 \mathrm{hpi}$, the infection medium was replaced with fresh medium. At $24 \mathrm{hpi}$, cells were harvested for luciferase assay or immunohistochemistry analysis. For liver and lung organoids, organoids were seeded in 24-well plates, pseudo-typed virus was added for MOI=0.01 and centrifuged the plate at 1200g, 1 hour. At $24 \mathrm{hpi}$, organoids were fixed for immunohistochemistry or harvested for luciferase assay following the Luciferase Assay System protocol (E1501, Promega)

\section{Single cell sequencing of hPSC-derived lung cells}

Single-cell capture, reverse transcription, cell lysis, and library preparation was performed using the Single Cell 3' version 3 kit and chip according to the manufacturer's protocol (10x Genomics, USA). Single-cell suspensions were generated by dissociating the cultured RUES2 cells with $0.05 \%$ Trypsin/0.02\% EDTA for 10-15 min, followed with passing through 40 $\mu \mathrm{M}$ strainer. The single cell suspension was achieved through sorting the dissociated cells in flow cytometry singlets. Cell count was adjusted to 1000-2000 cells per ul to target an estimated capture of 8000 cells. Six input wells were used. Sequencing was performed on NovaSeq6000 with setting 28 for read 1 and 91 for read 2. The sequencing data were primarily analyzed by CellRanger pipeline v3.0.2 (10x Genomics, USA). In particular, raw fastq data were generated by CellRanger mkfastq; A custom reference genome was built by integrating the virus and luciferase sequences into the 10x pre-built human reference (GRCh38 v3.0.0) using CellRanger mkref. Alignment of the raw reads to the custom reference genome, removing duplicated transcripts using the unique molecular identifiers (UMIs) and assignment to single cells was performed using CellRanger count.

Briefly, we used cells Seurat 3.1.4 R package for data analysis and visualization (Butler et al., 2018). The Seurat object is required at least 200 and at most 6000 unique molecular identifiers (UMIs), genes detected (UMI count >0) in less than two cells were removed. In addition, cells were excluded if more than $10 \%$ of sequences mapped to mitochondrial genes. In total, 5,080 cells from the sample passed these filters for quality.

Following the package suggestions, we used a linear model to mitigate the variation stemming from the number of detected unique molecules per cell. The differentially expressed genes were found by "vst" method and the top 3,000 differentially expressed genes were selected for PCA analysis. We used an elbow plot to determine the number of PCs. 20 PCs were used for each group of cells. Clustering resolution was set at 0.2 . For co-culture analysis, Macrophages and lung cells were re-clustered and re-analyzed, respectively. Macrophages were integrated using the first 20 dimensions of PCs and clustering resolution was set at 0.1. UMAP plots, heatmaps, violin plots and dotplots were generated by the Seurat toolkit FeaturePlot, DoHeatmap, VInPlot amd DotPlot functions, 
respectively. Cell types were determined using a combination of marker genes identified from the literature and the web-based tool Topp CellAtlas (https://toppgene.cchmc.org/)

\section{QUANTIFICATION AND STATSTICAL ANALYSIS}

Sample sizes for all figures and tables were estimated based on our previous studies(Chen et al., 2019; Huang et al., 2015; Huang et al., 2014; Yuling Han, 2020). For each set of experiments, samples were prepared for all experimental arms at the same time. All statistical tests are 2-sided. No adjustments were made for multiple comparisons. The relevant investigators (FD, LG and LY) were blinded to experimental allocations among different experimental arms for all experiments. $\mathrm{N}=3$ independent biological replicates were used for all experiments unless otherwise indicated. n.s. indicates a non-significant difference. For all parametric statistical analyses, data were determined to be normally distributed by the D'Agostino-Pearson test. For all parametric and non-parametric tests, variances were similar between groups being compared. For comparison between experimental and control groups at a specific time point or tissue site, 2-sided Student t- tests, one-way or two-way ANOVA test, chi-squared tests and two sided KolmogorovSmirnov test were used. All cells (RUES2, H1, HEK293T, THP-1, U937) were purchased from ATCC or WiCell in the past 2 years and were negative for mycoplasma. The hESC lines were regularly checked for chromosome abnormalities and maintained with normal chromosome numbers.

\section{Declarations}

\section{Author Contributions}

FD, LG, LY, YH, BN, PW, ZZ, CYM, TZ, XD, XW and DX performed the experiments and data analyses. All authors participated in the design and interpretation of some or all experiments. HJC, FD, and QZL wrote the manuscript, and all authors suggested editorial changes. HJC, JN and QZL conceived the study and recruited the collaborating partners.

We thank the other members in Huanhuan Joyce Chen lab (University of Chicago), Jie Na (Tsinghua University), Shuibing Chen (Weill Cornell Medicine), Todd Evans (Weill Cornell Medicine), and the Varmus Laboratory (Weill Cornell Medicine) for technical support. This work was supported by the National Key R\&D Program of China Grant 2017YFA0102802, 2019YFA0110001; U.S. National Institute of Health (1K99 CA226353-01A1); National Natural Science Grant of China (No 31571407);Hong Kong Health and Medical Research Fund (HMRF), Hong Kong (No:06172956).

\section{DATA AVAILABILITY}

scRNA-seq data is available from the GEO repository database with accession number GSE150708 (hPSC-derived lung cells, Coculture of macrophage and lung cells derived from hPSC, Co-culture of macrophage and lung cells derived from hPSC in SARS-CoV-2 infection).

Competing Financial Interests The authors have no financial conflicts of interest.

\section{References}

1. https://google.org/crisisresponse/covid19-map.

2. Allard, B., Panariti, A., and Martin, J.G. (2018). Alveolar Macrophages in the Resolution of Inflammation, Tissue Repair, and Tolerance to Infection. Frontiers in immunology 9, 1777.

3. Bian, Z., Gong, Y., Huang, T., Lee, C.Z.W., Bian, L., Bai, Z., Shi, H., Zeng, Y., Liu, C., He, J., et al. (2020). Deciphering human macrophage development at single-cell resolution. Nature May 20.

4. Buchrieser, J., James, W., and Moore, M.D. (2017). Human Induced Pluripotent Stem Cell-Derived Macrophages Share Ontogeny with MYB-Independent Tissue-Resident Macrophages. Stem cell reports 8, 334-345.

5. Cao, X., Yakala, G.K., van den Hil, F.E., Cochrane, A., Mummery, C.L., and Orlova, V.V. (2019). Differentiation and Functional Comparison of Monocytes and Macrophages from hiPSCs with Peripheral Blood Derivatives. Stem cell reports 12, 1282-1297.

6. Chan, M.W.Y., and Viswanathan, S. (2019). Recent progress on developing exogenous monocyte/macrophage-based therapies for inflammatory and degenerative diseases. Cytotherapy 21,393-415. 
7. Chen, H.J., Poran, A., Unni, A.M., Huang, S.X., Elemento, O., Snoeck, H.W., and Varmus, H. (2019). Generation of pulmonary neuroendocrine cells and SCLC-like tumors from human embryonic stem cells. The Journal of experimental medicine $216,674-$ 687.

8. Conti, P., Ronconi, G., Caraffa, A., Gallenga, C.E., Ross, R., Frydas, I., and Kritas, S.K. (2020). Induction of pro-inflammatory cytokines (IL-1 and IL-6) and lung inflammation by Coronavirus-19 (COVI-19 or SARS-CoV-2): anti-inflammatory strategies. Journal of biological regulators and homeostatic agents 34 .

9. Duan, F., Huang, R., Zhang, F., Zhu, Y., Wang, L., Chen, X., Bai, L., Guo, W., Chang, S.C., Hu, X., et al. (2018). Biphasic modulation of insulin signaling enables highly efficient hematopoietic differentiation from human pluripotent stem cells. Stem cell research \& therapy 9, 205.

10. Dye, B.R., Hill, D.R., Ferguson, M.A., Tsai, Y.H., Nagy, M.S., Dyal, R., Wells, J.M., Mayhew, C.N., Nattiv, R., Klein, O.D., et al. (2015). In vitro generation of human pluripotent stem cell derived lung organoids. eLife 4.

11. Gomez Perdiguero, E., Klapproth, K., Schulz, C., Busch, K., Azzoni, E., Crozet, L., Garner, H., Trouillet, C., de Bruijn, M.F., Geissmann, F., et al. (2015). Tissue-resident macrophages originate from yolk-sac-derived erythro-myeloid progenitors. Nature 518, $547-551$.

12. Guan, W.J., Ni, Z.Y., Hu, Y., Liang, W.H., Ou, C.Q., He, J.X., Liu, L., Shan, H., Lei, C.L., Hui, D.S.C., et al. (2020). Clinical Characteristics of Coronavirus Disease 2019 in China. The New England journal of medicine 382, 1708-1720.

13. Hoffmann, M.e.a. (2020). SARS-CoV-2 Cell Entry Depends on ACE2 and TMPRSS2 and Is Blocked by a Clinically Proven Protease Inhibitor. Cell, 16;181(182):271-280.e188. .

14. Huang, S.X., Green, M.D., de Carvalho, A.T., Mumau, M., Chen, Y.W., D'Souza, S.L., and Snoeck, H.W. (2015). The in vitro generation of lung and airway progenitor cells from human pluripotent stem cells. Nature protocols 10,413-425.

15. Huang, S.X., Islam, M.N., O'Neill, J., Hu, Z., Yang, Y.G., Chen, Y.W., Mumau, M., Green, M.D., Vunjak-Novakovic, G., Bhattacharya, J., et al. (2014). Efficient generation of lung and airway epithelial cells from human pluripotent stem cells. Nature biotechnology 32 , 84-91.

16. Hurley, K., Ding, J., Villacorta-Martin, C., Herriges, M.J., Jacob, A., Vedaie, M., Alysandratos, K.D., Sun, Y.L., Lin, C., Werder, R.B., et al. (2020). Reconstructed Single-Cell Fate Trajectories Define Lineage Plasticity Windows during Differentiation of Human PSCDerived Distal Lung Progenitors. Cell stem cell 26, 593-608 e598.

17. Jobe, O., Kim, J., Tycksen, E., Onkar, S., Michael, N.L., Alving, C.R., and Rao, M. (2017). Human Primary Macrophages Derived In Vitro from Circulating Monocytes Comprise Adherent and Non-Adherent Subsets with Differential Expression of Siglec-1 and CD4 and Permissiveness to HIV-1 Infection. Frontiers in immunology 8, 1352.

18. Kim, Y.I., Kim, S.G., Kim, S.M., Kim, E.H., Park, S.J., Yu, K.M., Chang, J.H., Kim, E.J., Lee, S., Casel, M.A.B., et al. (2020). Infection and Rapid Transmission of SARS-CoV-2 in Ferrets. Cell host \& microbe 27, 704-709 e702.

19. Kyle J. Travaglini, A.N.N., Lolita Penland, Stephen R. Quake, Mark A. Krasnow (2020). A molecular cell atlas of the human lung from single cell RNA sequencing. BioRXIV.

20. Lachmann, N., Ackermann, M., Frenzel, E., Liebhaber, S., Brennig, S., Happle, C., Hoffmann, D., Klimenkova, O., Luttge, D., Buchegger, T., et al. (2015). Large-scale hematopoietic differentiation of human induced pluripotent stem cells provides granulocytes or macrophages for cell replacement therapies. Stem cell reports 4, 282-296.

21. Lang, J., Cheng, Y., Rolfe, A., Hammack, C., Vera, D., Kyle, K., Wang, J., Meissner, T.B., Ren, Y., Cowan, C., et al. (2018). An hPSCDerived Tissue-Resident Macrophage Model Reveals Differential Responses of Macrophages to ZIKV and DENV Infection. Stem cell reports 11, 348-362.

22. Liao, M., Liu, Y., Yuan, J., Wen, Y., Xu, G., Zhao, J., Cheng, L., Li, J., Wang, X., Wang, F., et al. (2020). Single-cell landscape of bronchoalveolar immune cells in patients with COVID-19. Nature medicine.

23. Long Chen, J.X., Lei Bao, Yuan Shi (2020). Convalescent plasma as a potential therapy for COVID-19. the Lancet.

24. McCray, P.B., Jr., Pewe, L., Wohlford-Lenane, C., Hickey, M., Manzel, L., Shi, L., Netland, J., Jia, H.P., Halabi, C., Sigmund, C.D., et al. (2007). Lethal infection of K18-hACE2 mice infected with severe acute respiratory syndrome coronavirus. Journal of virology 81, 813-821.

25. Mehta, P., McAuley, D.F., Brown, M., Sanchez, E., Tattersall, R.S., Manson, J.J., and Hlh Across Speciality Collaboration, U.K. (2020). COVID-19: consider cytokine storm syndromes and immunosuppression. Lancet 395, 1033-1034.

26. Mills, C.D. (2015). Anatomy of a discovery: $\mathrm{m} 1$ and $\mathrm{m} 2$ macrophages. Frontiers in immunology 6, 212. 
27. Moroni, F., Dwyer, B.J., Graham, C., Pass, C., Bailey, L., Ritchie, L., Mitchell, D., Glover, A., Laurie, A., Doig, S., et al. (2019). Safety profile of autologous macrophage therapy for liver cirrhosis. Nature medicine 25, 1560-1565.

28. Mou, H., Zhao, R., Sherwood, R., Ahfeldt, T., Lapey, A., Wain, J., Sicilian, L., Izvolsky, K., Musunuru, K., Cowan, C., et al. (2012). Generation of multipotent lung and airway progenitors from mouse ESCs and patient-specific cystic fibrosis iPSCs. Cell stem cell 10, 385-397.

29. Murray, P.J. (2017). Macrophage Polarization. Annual review of physiology 79, 541-566.

30. Nie, J., Li, Q., Wu, J., Zhao, C., Hao, H., Liu, H., Zhang, L., Nie, L., Qin, H., Wang, M., et al. (2020). Establishment and validation of a pseudovirus neutralization assay for SARS-CoV-2. Emerg Microbes Infect 9, 680-686.

31. Rodell, C.B., Koch, P.D., and Weissleder, R. (2019). Screening for new macrophage therapeutics. Theranostics 9, 7714-7729.

32. Shapouri-Moghaddam, A., Mohammadian, S., Vazini, H., Taghadosi, M., Esmaeili, S.A., Mardani, F., Seifi, B., Mohammadi, A., Afshari, J.T., and Sahebkar, A. (2018). Macrophage plasticity, polarization, and function in health and disease. Journal of cellular physiology 233, 6425-6440.

33. Shi, Y., Wang, Y., Shao, C., Huang, J., Gan, J., Huang, X., Bucci, E., Piacentini, M., Ippolito, G., and Melino, G. (2020). COVID-19 infection: the perspectives on immune responses. Cell death and differentiation 27, 1451-1454.

34. Tay, M.Z., Poh, C.M., Renia, L., MacAry, P.A., and Ng, L.F.P. (2020). The trinity of COVID-19: immunity, inflammation and intervention. Nature reviews Immunology.

35. Wang, D., Hu, B., Hu, C., Zhu, F., Liu, X., Zhang, J., Wang, B., Xiang, H., Cheng, Z., Xiong, Y., et al. (2020). Clinical Characteristics of 138 Hospitalized Patients With 2019 Novel Coronavirus-Infected Pneumonia in Wuhan, China. Jama.

36. Wenhui Li, e.a. (2003). Angiotensin-converting enzyme 2 is a functional receptor for the SARS coronavirus. Nature 426, pages 450-454.

37. Whitt, M.A. (2010). Generation of VSV pseudotypes using recombinant DeltaG-VSV for studies on virus entry, identification of entry inhibitors, and immune responses to vaccines. J Virol Methods 169, 365-374.

38. Wynn, T.A., Chawla, A., and Pollard, J.W. (2013). Macrophage biology in development, homeostasis and disease. Nature 496, 445-455.

39. Xu, Z., Shi, L., Wang, Y., Zhang, J., Huang, L., Zhang, C., Liu, S., Zhao, P., Liu, H., Zhu, L., et al. (2020). Pathological findings of COVID-19 associated with acute respiratory distress syndrome. The Lancet Respiratory medicine 8, 420-422.

40. Yuling Han, F.D., Lewis Cantley, Benjamin tenOever, David Ho, Todd Evans, Robert E. Schwartz, Huanhuan Joyce Chen, Shuibing Chen. et.al (2020). Identification of Candidate COVID-19 Therapeutics using hPSC-derived Lung Organoids. BioRxiv.

41. Ziegler, C.G.K., Allon, S.J., Nyquist, S.K., Shalek, A.K., Ordovas-montanes, J., and Lung, H.C.A. (2020). SARS-CoV-2 Receptor ACE2 Is an Interferon- Stimulated Gene in Human Airway Epithelial Cells and Is Detected in Specific Cell Subsets across Tissues. Cell 27 April.

\section{Figures}




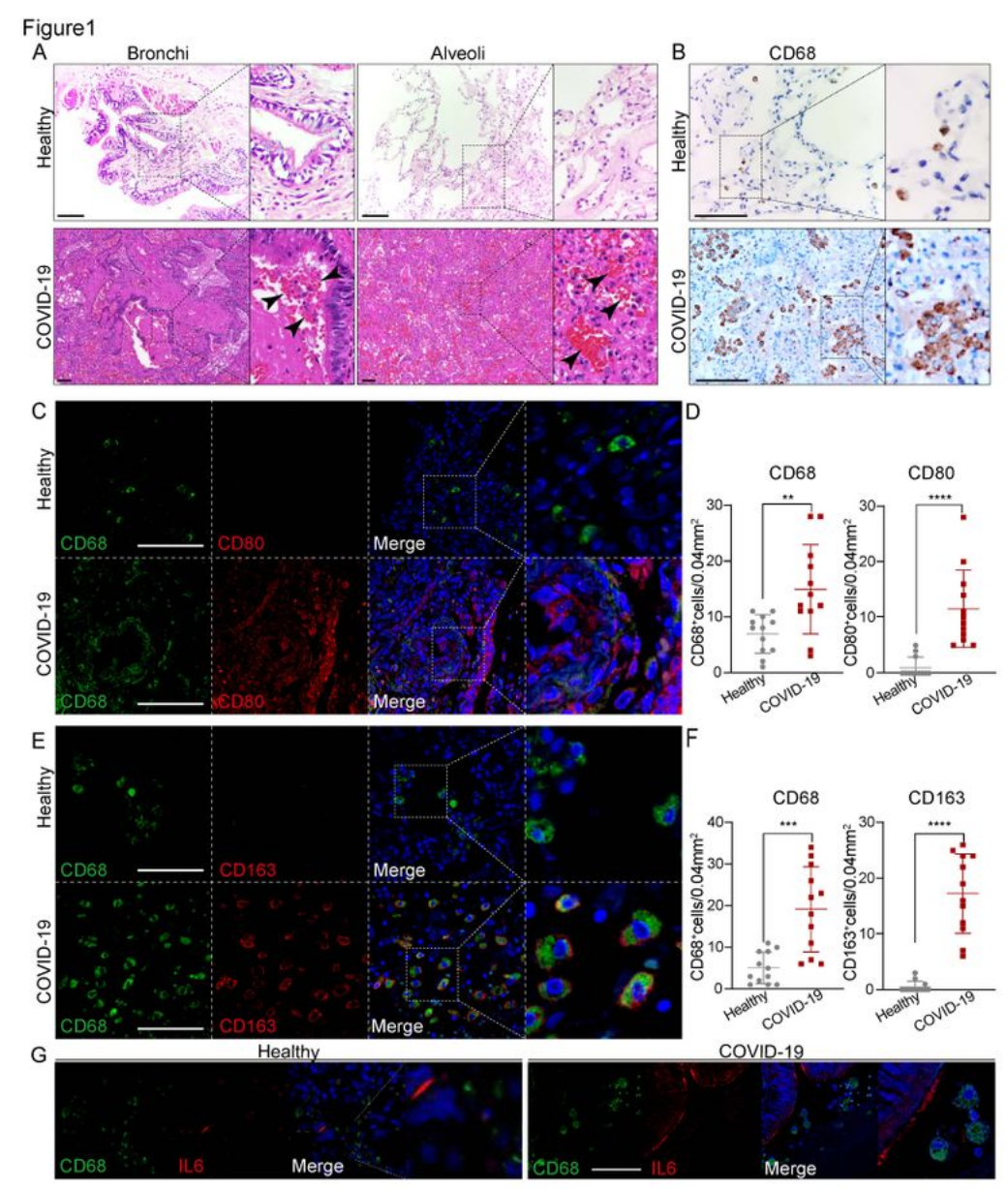

\section{Figure 1}

Macrophages were highly involved at the severe stage of COVID-19 (A) H+E (Hematoxylin and Eosin) staining on the bronchial or alveolar region in healthy or severe COVID-19 case. Pulmonary hemorrhagic infarct (denoted by arrow heads) (B) Immunohistochemistry $(\mathrm{IHC})$ using antibody against CD68 revealed macrophage with aggregated phenotype and enlarged nuclei in COVID-19 lung, compared to the ones in healthy lung. (C) Immunofluorescence (IF) staining on healthy or COVID-19 distal lung tissues using antibodies against CD68 (pan-macrophage marker), and CD80 (M1 macrophage marker) (D) Quantification on CD68+ or CD80+ macrophages in healthy or COVID-19 distal lung tissues. (E) IF staining on healthy or COVID distal lung tissues using antibodies against CD68 and CD163 (M2 macrophage marker) (F) Quantification on CD68+ or CD163+ macrophages in healthy or COVID-19 distal lung tissues. (G) IF staining on healthy or COVID-19 distal lung tissues using antibodies against CD68 and IL-6. Scale bar $=100 \mu \mathrm{m}$ in all images in Figure 1. Data was presented as mean \pm STDEV. P values were calculated by unpaired two-tailed Student's $t$ test. ${ }^{\star *} \mathrm{P}<0.01,{ }^{\star \star *} \mathrm{P}<0.001$, and ${ }^{\star \star \star *} \mathrm{P}<0.0001$. 

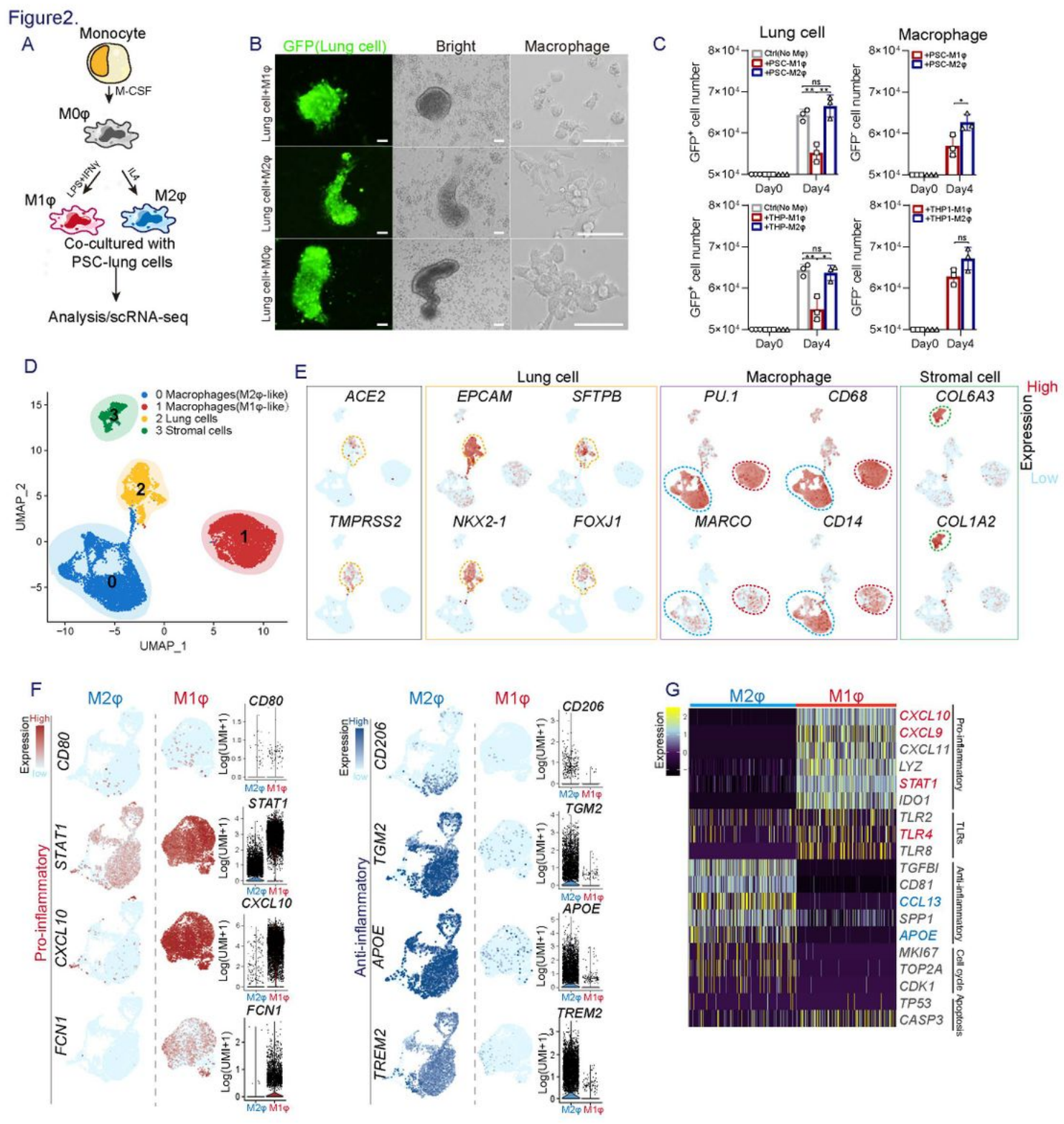

\section{Figure 2}

Characterization of the co-culture of lung cells and macrophages derived from hPSCs (A) Schematic of the experimental flowchart on the co-cultures. (B) Representative bright or fluorescence images of the co-culture of lung cells and macrophages derived from hPSC line RUES2. Lung cells are GFP positive. Scale bar $=50 \mu \mathrm{m}$ (C) Quantification of lung cells, and macrophages in the co-cultures of lung cells and $\mathrm{iM} 1 \varphi, \mathrm{iM} 2 \varphi$ or $293 \mathrm{~T}$ cells. $\mathrm{P}$ values were calculated by unpaired two-tailed Student's $\mathrm{t}$ test. ${ }^{*} \mathrm{P}<0.05$, ${ }^{\star *} \mathrm{P}<0.01$. (D) UMAP of scRNA seq on the two-co-cultures (lung and iM1 $\varphi$ co-culture; lung and $\mathrm{iM} 2 \varphi$ co-culture). Colored and annotated with cluster 0-3 representing $\mathrm{iM} 2 \varphi$, iM1 $\varphi$, lung epithelial cells and stromal cells. (E) ACE2, TMPRSS2, as well as putative cell-fate related markers differentially expressed in each cluster in UMAPs. Relative expression of each marker gene range from low (light blue) to high (pink) as indicated. Individual cell positive for each marker are donated by red dots. The main population of ACE2 or TMPRSS2 positive populations are circled in dotted line. (F) A set of pro-or anti-inflammatory factors, or cell-fate related markers differentially expressed in the cluster of $\mathrm{iM} 1 \varphi$ or $\mathrm{iM} 2 \varphi$ in UMAPs. Relative expression of each marker gene range from low (light blue) to high (pink) as indicated. The violin plot shows the expression level (log2(UMl+1)) of indicated gene in each cluster. (G) Heatmap presenting top differential expression genes related to pro- or anti-inflammatory factors, Toll like receptors (TLRs), cell cycle regulation or apoptosis, in $\mathrm{iM} 2 \varphi$ or $\mathrm{iM} 1 \varphi$. 
Figure 3
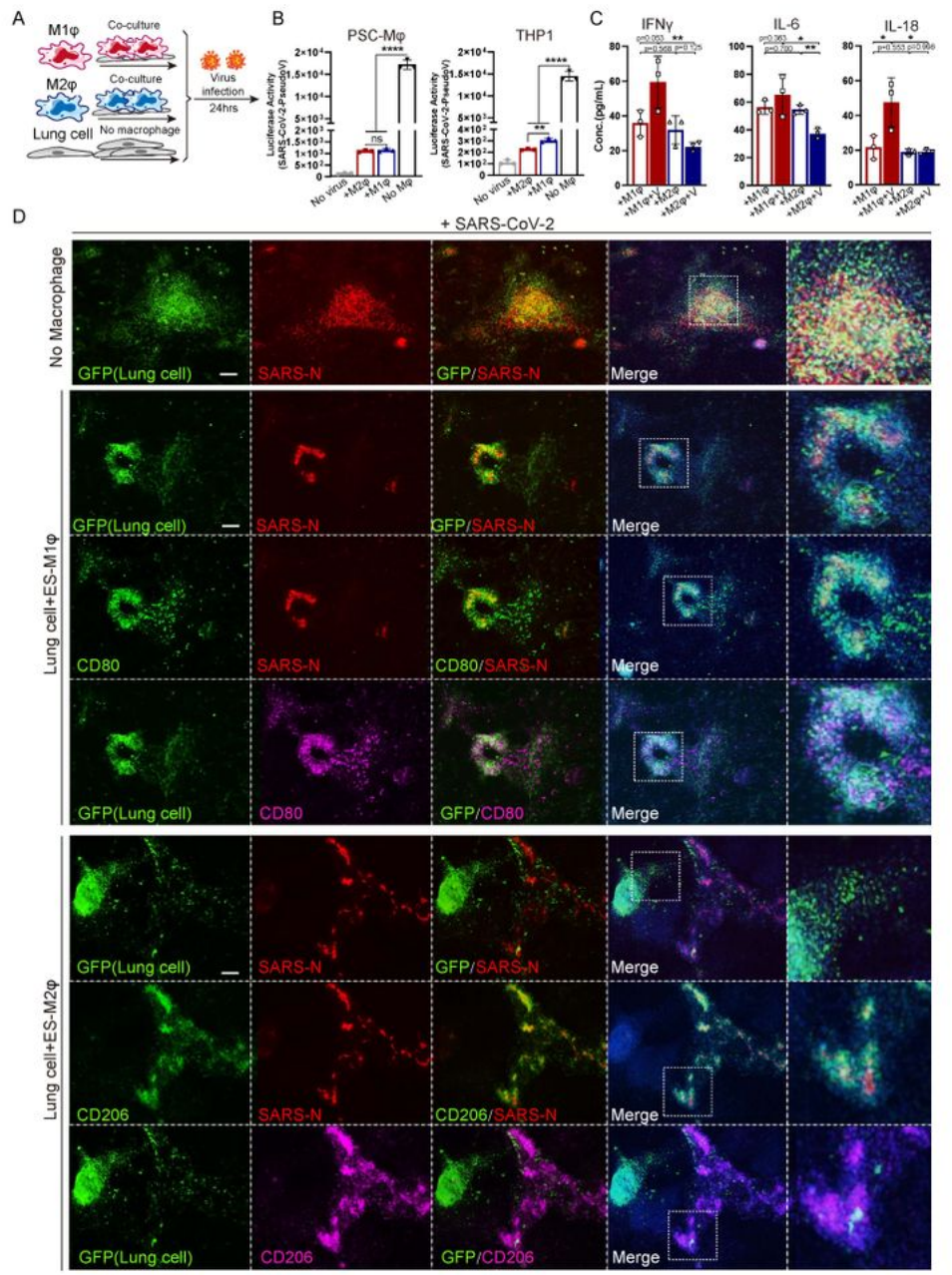

\section{Figure 3}

The effects of M1 or M2 macrophages on SARS-CoV-2 infection (A) Schematic of the experimental flowchart on the co-cultures. (B) Luciferase activity of the co-cultures of lung cells and M1, M2 macrophages (iM $\varphi$ or THP-1) or 293T cells (control) at Mock or infected with SARS-CoV-2 pseudo-entry virus at $24 \mathrm{hpi} \mathrm{(MOI=0.01).} \mathrm{P} \mathrm{values} \mathrm{were} \mathrm{calculated} \mathrm{by} \mathrm{unpaired} \mathrm{two-tailed} \mathrm{Student's} t$ test. $\star \star P<0.01$, $* \star \star \star P<0.0001$. (C) Quantification of inflammatory factors in the co-culture medium at Mock or infected with SARS-CoV-2 pseudo-entry virus at $24 \mathrm{hpi}(\mathrm{MOI}=0.01)$. $\mathrm{P}$ values were calculated by Chi-Square Test. ${ }^{\star} \mathrm{P}<0.5,{ }^{\star *} \mathrm{P}<0.01$. (D) IF staining on the cocultures of iLung cells and $\mathrm{iM} 1 \varphi$, iM2 $\varphi$, or 293T, at Mock or infected with SARS-CoV-2 virus at $24 \mathrm{hpi}(\mathrm{MOl}=0.01)$, using antibodies detecting SARS-N protein, CD80 or CD206. ILung cells expressed GFP. Scale bar $=100 \mu \mathrm{m}$ 


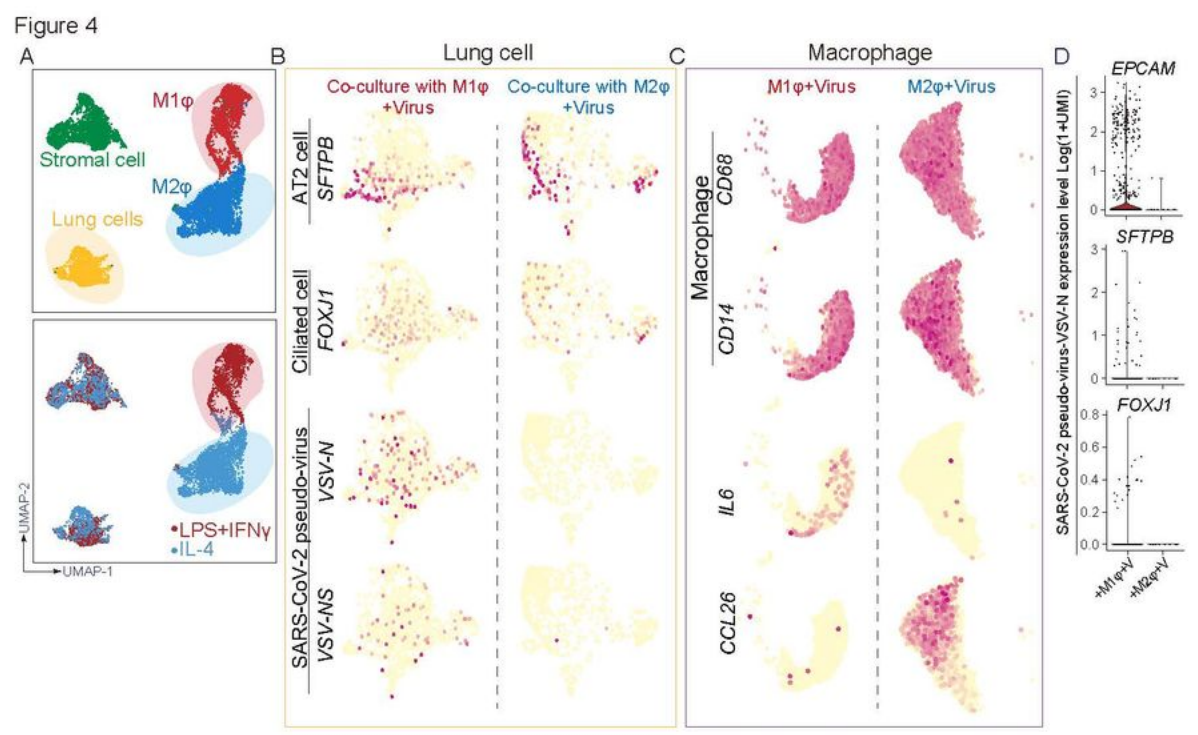

E

GO:Enrichment in $\mathbf{M} 1 \varphi$ (M1 $\varphi+$ virus)

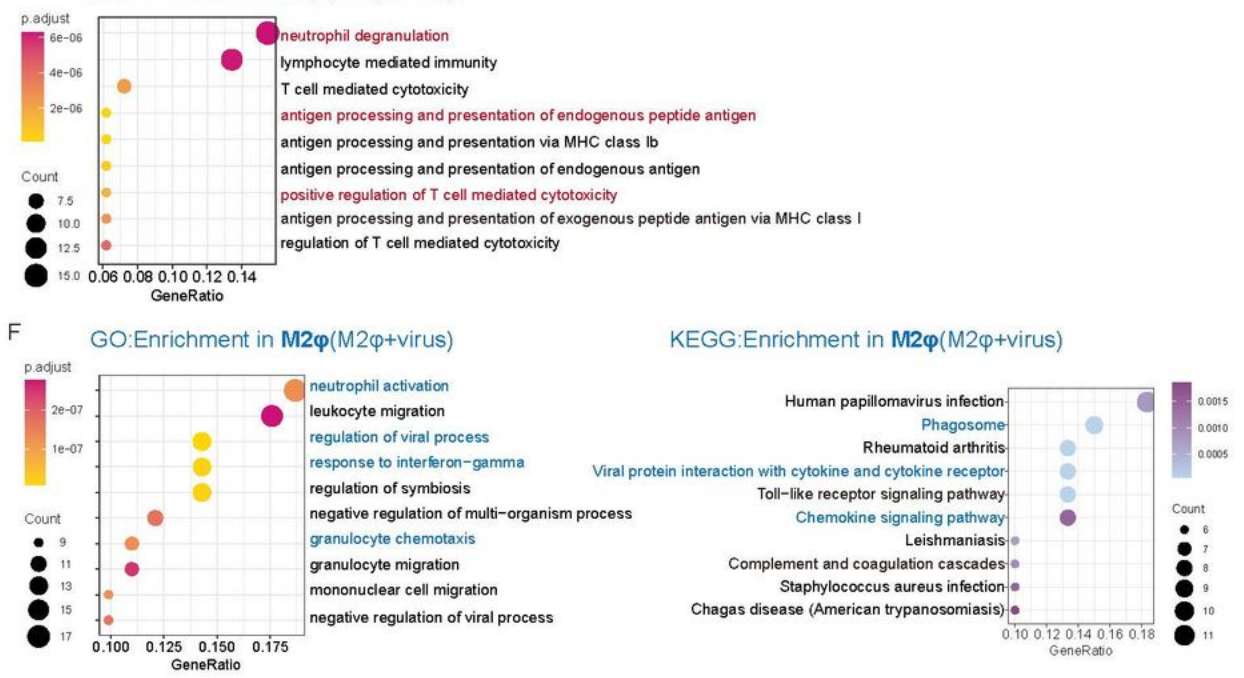

\section{Figure 4}

scRNA analysis of $\mathrm{iM} 1 \varphi$, iM2 $\varphi$ or iLung upon viral infection (A) UMAP of scRNA seq on the SARS-CoV-2 pseudo-virus infected cocultures (lung and $\mathrm{iM} 1 \varphi$ co-culture; lung and $\mathrm{iM} 2 \varphi$ co-culture). Colored and annotated with 4 clusters representing $\mathrm{iM} 2 \varphi$, iM $1 \varphi$, lung cells and stromal cells. (B) SARS-CoV-2 pseudo-virus genes, and putative cell-fate related markers differentially expressed in iLung cells co-cultured with $\mathrm{iM} 1 \varphi$ or iM2 $\varphi$ in UMAPs. Relative expression of each marker gene range from low (light yellow) to high (red) as indicated. Individual cell positive for each marker are denoted by red dots. (C) Inflammatory factors (IL6 and CCL26), and putative cell-fate related markers(CD14 and CD68) differentially expressed in $\mathrm{iM} 1 \varphi$ or $\mathrm{iM} 2 \varphi$ co-cultured with ilung in UMAPs. Relative expression of each marker gene range from low (light yellow) to high (red) as indicated. Individual cell positive for each marker are denoted by red dots. (D) The violin plot shows the expression level (log2(UMI+1)) of SARS-CoV-2 pseudo-virus specific genes in each cluster. (E) GO enrichment analysis in $\mathrm{iM} 1 \varphi$. Important pathways related to immune response or viral reaction is highlighted in red $(\mathrm{iM} 1 \varphi)(\mathrm{F}) \mathrm{GO}$ and KEGG enrichment analysis in $\mathrm{iM} 2 \varphi$. Important pathways related to immune response or viral reaction is highlighted in blue (iM2 $\varphi)$ 


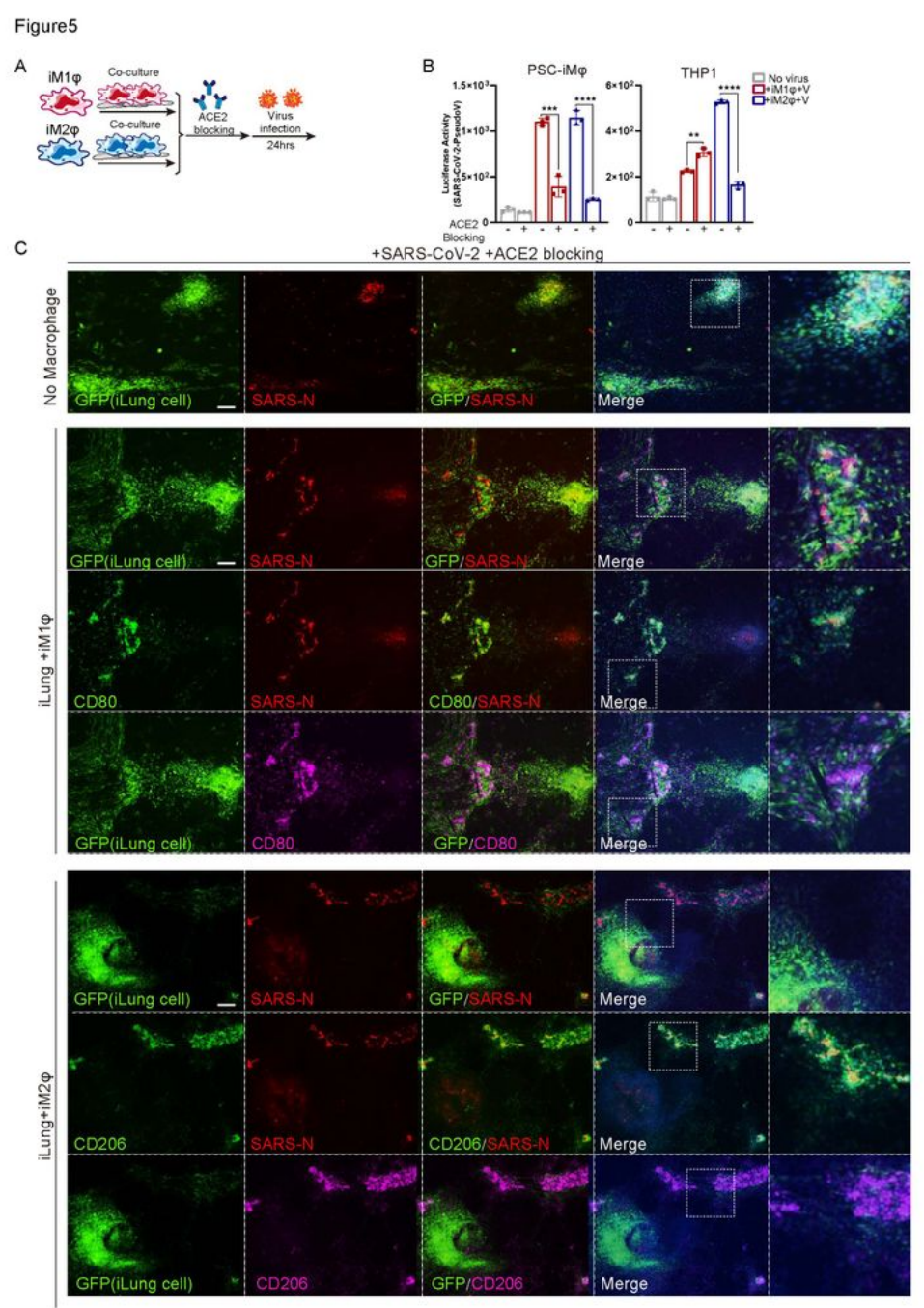

\section{Figure 5}

The effects of macrophages in combination with ACE2 blockage on SARS-CoV-2 infection (A) Schematic of the experimental flowchart on the co-cultures. (B) The ACE2 blockage antibody was applied two hours prior to the virus presence, and the luciferase activity of the co-cultures of lung cells and M1, M2 macrophages (iM $\varphi$ or THP-1) or 293T cells (control) was measured at Mock or infected with SARS-CoV-2 pseudo-entry virus at $24 \mathrm{hpi}(\mathrm{MOI}=0.01)$. P values were calculated by unpaired two-tailed Student's $\mathrm{t}$ test. $\star \star \star P<0.001, * \star \star \star P<0.0001$. (C) The ACE2 blockage antibody was applied two hours prior to the virus presence, IF staining was performed on the co-cultures of iLung cells and $\mathrm{iM} 1 \varphi$, iM2 $\varphi$, or 293T, at Mock or infected with SARS-CoV-2 virus at $24 \mathrm{hpi}(\mathrm{MOI}=0.01)$, using antibodies detecting SARS-CoV-2 NSP14 protein, CD80 or CD206. ILung cells expressed GFP. Scale bar $=100 \mu \mathrm{m}$

\section{Supplementary Files}

This is a list of supplementary files associated with this preprint. Click to download.

- FigureS1.jpg

- FigureS2.jpg

- FigureS3.jpg 
- FigureS4.jpg

- FigureS5.jpg

- FigureS6.jpg

- FigureS7.jpg

- FigureS8.jpg

- FigureS9.jpg 